\title{
Recoil-induced dissociation in hard-x-ray photoionization
}

\author{
Ji-Cai Liu, ${ }^{1,2, *}$ Vinícius Vaz da Cruz $\odot,{ }^{3, *}$ Sergey Polyutov, ${ }^{4,5}$ Alexander Föhlisch, ${ }^{3,6}$ and Faris Gel'mukhanov ${ }^{4,5,7}$ \\ ${ }^{1}$ Department of Mathematics and Physics, North China Electric Power University, 102206 Beijing, China \\ ${ }^{2}$ Collaborative Innovation Center of Light Manipulations and Applications, Shandong Normal University, Jinan 250358, China \\ ${ }^{3}$ Institute of Physics and Astronomy, University of Potsdam, Karl-Liebknecht-Strasse 24-25, 14476 Potsdam, Germany \\ ${ }^{4}$ Siberian Federal University, 660041 Krasnoyarsk, Russia \\ ${ }^{5}$ Kirensky Institute of Physics, Federal Research Center KSC SB RAS, 660036 Krasnoyarsk, Russia \\ ${ }^{6}$ Institute for Methods and Instrumentation in Synchrotron Radiation Research FG-ISRR, Helmholtz-Zentrum Berlin \\ für Materialien und Energie Albert-Einstein-Strasse 15, 12489 Berlin, Germany \\ ${ }^{7}$ Theoretical Chemistry \& Biology, Royal Institute of Technology, S-106 91 Stockholm, Sweden
}

(Received 8 August 2019; published 15 November 2019)

\begin{abstract}
We predict the recoil-induced molecular dissociation in hard-x-ray photoionization. The recoil effect is caused by electronic and photon momentum exchange with the molecule. We show the strong role of relativistic effects for the studied molecular fragmentation. The recoil-induced fragmentation of the molecule is caused by elongation of the bond due to the vibrational recoil effect and because of the centrifugal force caused by the rotational recoil. The calculations of the x-ray photoelectron spectra of the $\mathrm{H}_{2}$ and NO molecules show that the predicted effects can be observed in high-energy synchrotrons like SOLEIL, SPring-8, PETRA, and XFEL SACLA. The relativistic effect enhances the recoil momentum transfer and makes it strongly sensitive to the direction of ejection of the fast photoelectron with respect to the photon momentum.
\end{abstract}

DOI: 10.1103/PhysRevA.100.053408

\section{INTRODUCTION}

The dynamics and spectroscopy of highly excited states of molecules is an issue of great importance to chemical physics. The photon recoil effect is used in laser physics for optical cooling and deflection of atoms and molecules [1] with important applications to fundamental aspects of quantum mechanics such as Bose-Einstein condensation and atom interferometry. It is well known that photons [2] and photoelectrons [3] can transfer significant linear momentum as well as angular momentum in the course of $\mathrm{x}$-ray absorption, scattering, and ionization. The related recoil-induced vibrational and rotational excitations have received significant attention in x-ray photoelectron and Auger spectroscopies in the sub-keV x-ray energy range, where electronic recoil dominates and its role is rather weak. This generally justifies the use of the time-honored Franck-Condon (FC) principle in the soft-x-ray region where the momentum exchange between the photoelectron and molecules manifests itself as small rotational and translational Doppler broadenings as well as a small recoil shift of the vibrational resonances due to momentum transfer to the center-of-gravity (CG) of the molecule. In the last decade, the interest in this field has

\footnotetext{
*Corresponding authors: jicailiu@ ncepu.edu.cn; vazdacruz@uni-potsdam.de

Published by the American Physical Society under the terms of the Creative Commons Attribution 4.0 International license. Further distribution of this work must maintain attribution to the author(s) and the published article's title, journal citation, and DOI.
}

increased, mainly due to the available super-high spectral resolution in the photoelectron energy range below $10 \mathrm{keV}$ which allowed observation of recoil-induced vibrational excitation [4-8], translational and rotational recoil shifts [9], the rotational Doppler effect [10-14], and recoil-induced Doppler splitting $[10,15,16]$. The recoil shifts of the photoelectron lines were observed also in solids such as graphite [17], heavy fermion material $\mathrm{LiV}_{2} \mathrm{O}_{4}$ [18], and $\mathrm{Al}$ and Au metals [19].

However, the already existing synchrotron sources of x-ray radiation such as SOLEIL [20] and SPring-8 [17,21] deliver high brilliance synchrotron radiation up to $\sim 12 \mathrm{keV}$ energies. Hard-x-ray photoelectron spectra at excitation energies of $7940 \mathrm{eV}$ were measured with a resolution of about $100 \mathrm{meV}$ [17]. X-ray photons with an energy of $100-200 \mathrm{keV}$ are available at the PETRA III synchrotron [22,23]. The X-ray free-electron facility (XFEL) SACLA [24] generates x-ray radiation with photon energies up to $20 \mathrm{keV}$ and intensity $\sim 10^{20} \mathrm{~W} / \mathrm{cm}^{2}$, which allows us to overcome low ionization cross sections in the high-energy region. Such high-energy photons allow us to reach rovibrational states close to the dissociation limit and even to dissociate the molecule. One can reach the rotational states $J>100$ with an effective temperature of $10^{5}-10^{6} \mathrm{~K}$. It is important that light creates a highly coherent rovibrational nuclear wave packet which can be controlled by analyzing x-ray fluorescence or Auger spectra of core-ionized molecules [20,25-27] as well as optical spectroscopy $[28,29]$. Exquisite control over all the degrees of freedom of highly excited molecular cations with huge quantum numbers is a precursor for exploring the transitions between the quantum and classical world. Perturbative approaches do not work at such high levels of excitation, where coupling between degrees of freedom changes dramatically 


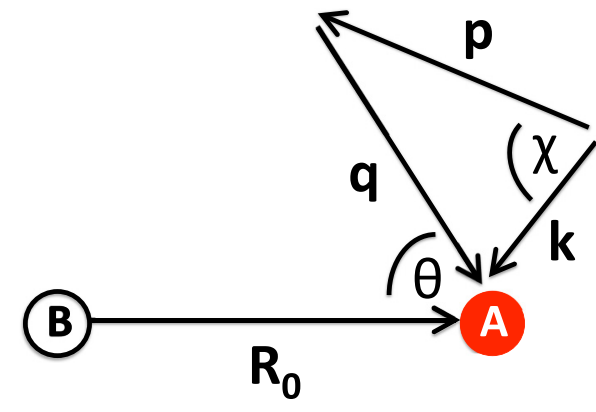

FIG. 1. Orientation of the momenta of the photon $\mathbf{k}$, the photoelectron $\mathbf{p}$, and $\mathbf{q}=\mathbf{k}-\mathbf{p}$ with respect to the initial internuclear radius vector $\mathbf{R}_{0}$.

from what is observed in the soft-x-ray region. As a result, interpreting molecular spectra becomes increasingly difficult as the level of excitation grows. Moreover, the underlying physical picture of the recoil effect in the region above 10 $\mathrm{keV}$ is unknown. Indeed, the photon recoil neglected in the sub-keV region starts to compete with the electronic recoil when we pass the $10-\mathrm{keV}$ energy range and the photoelectron becomes a relativistic object. Furthermore, the large recoil energy delivered to vibrations and rotations can break the chemical bond as we show here. This makes understanding $\mathrm{x}$-ray spectroscopy in the energy range above $10 \mathrm{keV}$ an ongoing challenge and very timely.

\section{THEORY}

Before we discuss the consequences of the recoil effects, we must first pay attention to two important points which we face in the hard-x-ray region. First of all, the photon momentum $(\mathbf{k}, k=\omega / c)$ starts to approach the electron momentum $\mathbf{p}$ when the energy of the photon $\omega$ approaches the rest energy of the electron $m c^{2} \approx 510.7 \mathrm{keV}$. The second important point is that now the speed of the photoelectron can be comparable with the speed of light, $c=137$ a.u. (we use atomic units: $m=\hbar=e=1)$. This requires us to treat the electron as a relativistic object. Both the photon and electron transfer to the molecule the recoil momentum (see Fig. 1)

$$
\mathbf{q}=\mathbf{k}-\mathbf{p}
$$

The kinetic energy of the relativistic electron $E=$ $\sqrt{p^{2} c^{2}+m^{2} c^{4}}-m c^{2}$ and the energy conservation law [30] $\omega=I+E$ allow us to compute $q=\sqrt{k^{2}+p^{2}-2 p k \cos \chi}$ :

$$
q=k \sqrt{(1-\beta)^{2}+4 \beta \sin ^{2} \frac{\chi}{2}} .
$$

Here $\beta=\sqrt{\Omega\left(\Omega+2 m c^{2}\right) / \omega^{2}}, \Omega=\omega-I, I$ is the ionization potential, and $\chi=\angle(\mathbf{p}, \mathbf{k})$. The recoil momentum $q \approx$ $p_{\mathrm{NR}}=\sqrt{2 m \Omega}$ coincides with the momentum of the nonrelativistic electron $p_{\mathrm{NR}}$ when $\omega \ll m c^{2}$ and $q \approx m c[1+$ $\left.2\left(\frac{\omega}{m c^{2}}\right)^{2} \sin ^{2} \frac{\chi}{2}\right]$ in ultrarelativistic region $\omega \gg m c^{2}$. Figure 2 shows that $p_{\mathrm{NR}}>k$ in the low-energy region while the photon momentum dominates in the high-energy region $k>p_{\mathrm{NR}}$. In contrast, the momentum of the relativistic photoelectron cannot be smaller than $k\left[p=\sqrt{\frac{\Omega}{c}\left(\frac{\Omega}{c}+2 m c\right)}>k\right]$ except for the tiny region near the ionization threshold, $\Omega<\omega k / 2 m c$.

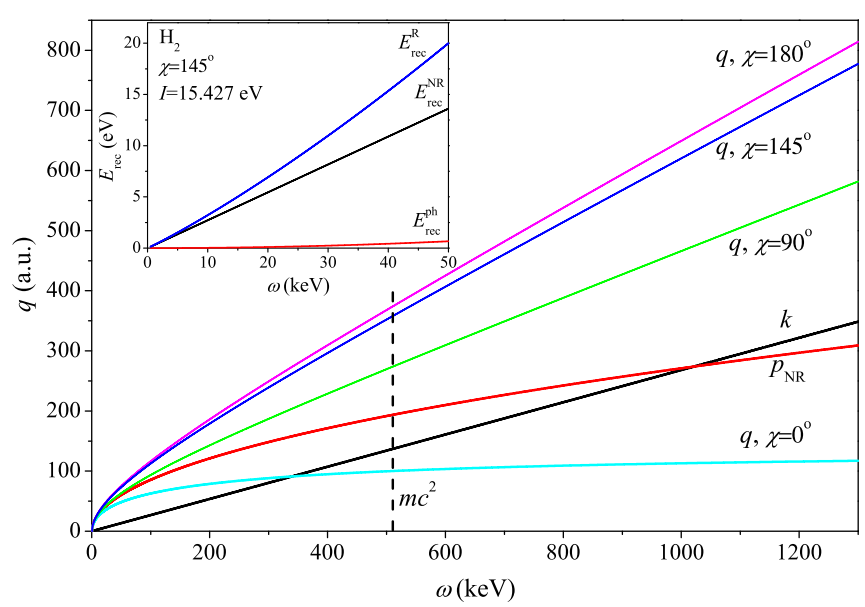

FIG. 2. The dependence of the recoil momentum $q$ on $\omega$ and $\chi=\angle(\mathbf{p}, \mathbf{k})$ compared with the dispersion of the nonrelativistic momentum of the photoelectron $p_{\mathrm{NR}}$ and the dispersion of the photon momentum $k$. The vertical line $\omega=m c^{2}=510.7 \mathrm{keV}$ separates very approximately the nonrelativistic and ultrarelativistic regions. The insert shows the recoil energy for the $\mathrm{H}_{2}$ molecule. One can see that the relativistic effect becomes important for $\mathrm{H}_{2}$ starting from $\omega=10 \mathrm{keV}$.

Figure 2 shows a strong dependence of $q$ on the direction of ejection of the photoelectron and very strong deviation of the dispersion law of $q$ from the dispersion of the nonrelativistic momentum of the photoelectron $p_{\mathrm{NR}}=\sqrt{2 m \Omega}$ as well as from the dispersion of the photon momentum $k=\omega / c$. We also reach the important conclusion that both the electron and the photon contribute equally to the recoil effect in the hard-x-ray region. Furthermore, one can see that the recoil momentum and, hence, the recoil energy $E_{\text {rec }} \propto q^{2}$, increases drastically with increase of the angle $\chi$ (Fig. 2).

The momentum exchange between the molecule and the photoelectron and photon affects the center-of-gravity (CG) of the molecule and internal vibrational and rotational motions. When the $\mathrm{x}$-ray photon is absorbed and the fast electron is subsequently ejected from atom $\mathrm{A}$ of a diatomic molecule AB with mass $M=M_{A}+M_{B}$, the center-of-gravity of the molecule gains the momentum q. This enlarges the kinetic energy of the center-of-gravity by the recoil energy

$$
E_{\text {rec }}^{\mathrm{CG}}=q^{2} m / 2 M .
$$

The internal vibrational motion acquires the momentum $\alpha q \cos \theta$ along the molecular axis, where $\theta$ is the angle between $\mathbf{q}$ and internuclear radius vector $\mathbf{R}=\mathbf{R}_{A}-\mathbf{R}_{B}, \alpha=$ $M_{B} / M$. The component of $\mathbf{q}$ orthogonal to $\mathbf{R}$ creates recoil angular momentum $\mathbf{J}=\alpha\left[\mathbf{R}_{0} \times \mathbf{q}\right]$ at the instant of the photoionization

$$
J=J(\theta)=\alpha q R_{0} \sin \theta,
$$

which happens at the ground-state equilibrium distance $R_{0}$ (see Fig. 1). Thus the vibrational and angular, or rotational, recoils enlarge the vibrational and rotational energies by the vibrational and rotational recoil energies $E_{\mathrm{rec}}^{\mathrm{vib}}(\theta)=E_{\mathrm{rec}} \cos ^{2} \theta$ and $E_{\mathrm{rec}}^{\mathrm{rot}}(\theta)=E_{\mathrm{rec}} \sin ^{2} \theta$, respectively. The total recoil energy

$$
E_{\mathrm{rec}}=E_{\mathrm{rec}}^{\mathrm{vib}}(\theta)+E_{\mathrm{rec}}^{\mathrm{rot}}(\theta)=\alpha^{2} q^{2} m / 2 \mu,
$$


transferred to the internal molecular motion is shared almost equally between vibrational and rotational degrees of freedom. Here $\mu=M_{A} M_{B} / M$ is the reduced mass. One should mention that the term "vibrational" is used to denote both the recoil-induced vibrations and dissociation induced by the recoil momentum $\alpha q \cos \theta$ along the molecular axis.

To include the recoil effects in the formalism one should abandon the FC approximation and include the electronic transition dipole moment $\mathbf{d} \propto \mathbf{q} \exp \left(\iota \mathbf{q} \cdot \mathbf{R}_{A}\right)=\mathbf{q} \exp (\iota \alpha \mathbf{q} \cdot \mathbf{R})$ of photoionization of an $s$ electron from the site A into the FC amplitude between initial and final rovibronic nuclear states characterized by the vibrational and rotational quantum numbers

$$
F_{0, \nu \mathbf{J}}=\left\langle\psi_{0}\left|e^{\imath \alpha q R \cos \theta}\right| \psi_{\nu, \mathbf{J}}\right\rangle .
$$

Following the standard procedure [25], one can write the expression for the ionization cross section with the FC amplitude (6)

$$
\begin{aligned}
\frac{d^{2} \sigma}{d O d E} & =\sigma_{\mathrm{el}}\left(E_{B}, \omega, \chi\right) P\left(E_{B}, \omega, \chi\right), \\
P\left(E_{B}, \omega, \chi\right) & =\int_{0}^{\pi} d \theta \sin \theta P\left(E_{B}, \omega, \chi, \theta\right), \\
P\left(E_{B}, \omega, \chi, \theta\right) & =\frac{1}{\pi} \operatorname{Re} \int_{0}^{\infty} d t e^{\left[\imath\left(E_{B}-I-E_{\mathrm{rec}}^{\mathrm{CG}}+\epsilon_{0}\right)-\Gamma\right] t} \sigma(t, \theta), \\
H_{i} & =-\frac{1}{2 \mu} \frac{\partial^{2}}{\partial R^{2}}+\frac{\hat{\mathbf{J}}^{2}}{2 \mu R^{2}}+V_{i}(R) .
\end{aligned}
$$

We use the time-dependent representation deliberately to describe on the same footing the bound and dissociative nuclear states. Here $|\psi(0)\rangle=e^{-l \alpha q R \cos \theta}\left|\psi_{0}\right\rangle,|\psi(t)\rangle=e^{-l H_{i} t}|\psi(0)\rangle$, $E_{B}=\omega-E$ is the binding energy, $\epsilon_{0}=\omega_{0} / 2$ is the zeropoint energy of the ground state, $P=\sum\left|F_{0 v}\right|^{2} \Delta\left(E_{B}-I-\right.$ $\left.\left(\epsilon_{v}-\epsilon_{0}\right), \Gamma\right)$ with $\Delta(E, \Gamma)=\Gamma / \pi\left(E^{2}+\Gamma^{2}\right), \int P d E=1$, and $V_{i}(R)$ is the potential energy of the ionized state with $\left[V_{i}(R)\right]_{\min }=0$. The photoelectron spectrum for angle $\theta$ is given by the half-Fourier transform of the autocorrelation function $\sigma(t, \theta)=\int_{0}^{\infty} d R \psi^{*}(0) \psi(t)$. The electronic cross section $\sigma_{\mathrm{el}}$ of $1 \mathrm{~s}$ ionization for a hydrogen-like atom can be computed using Eq. (57.8) from Ref. [30]. Here, we neglected the thermal rotational and translation motions in the ground state whose effect is rather small because we study rovibrational excitations with an effective temperature $\gtrsim 10^{4} \mathrm{~K}$, except, the translational and rotational Doppler broadenings [11] which we will discuss below.

In view of the fact that the recoil-induced angular momentum $J$ in the ionized state is large, one can replace the operator $\hat{\mathbf{J}}^{2}$ in the Hamiltonian by the square of the classical momentum $J^{2}(\theta)$ (4) according to the correspondence principle. This allows us to write the semiclassical Hamiltonian

$$
\begin{gathered}
H_{i} \approx-\frac{1}{2 \mu} \frac{\partial^{2}}{\partial R^{2}}+V_{i}(R, \theta), \\
V_{i}(R, \theta)=V_{i}(R)+E_{\mathrm{rec}}\left(\frac{R_{0}}{R}\right)^{2} \sin ^{2} \theta,
\end{gathered}
$$

where the rotational kinetic energy is included in the effective potential $V_{i}(R, \theta)=V_{i}(R)+J^{2}(\theta) /\left(2 \mu R^{2}\right)$ (see Fig. 3). The semiclassical approximation simplifies significantly the simulations and gives deep insight into the physics of vibrational and rotational dissociation.

\section{BOND BREAKING IN $\mathrm{H}_{2}$ AND NO MOLECULES}

We applied the developed theory to two showcase molecules: $\mathrm{H}_{2}$ and NO. In the simulations we used the Morse potential $V_{i}(R)=D_{i}\left(1-e^{-\zeta_{i}\left(R-R_{0}^{(i)}\right)}\right)^{2}$ with the parameters $\left(R_{0}^{(i)}, \omega_{0}^{(i)}, D_{i}, \zeta_{i}=\omega_{0}^{(i)} \sqrt{\mu / 2 D_{i}}\right)$ extracted from experimental data. $\mathrm{H}_{2} X^{1} \Sigma_{g}^{+}\left(\mathrm{H}_{2}^{+} X^{2} \Sigma_{g}^{+}\right)$[31,32]: $R_{0}=1.40189$ a.u. (2.00378 a.u.), $\omega_{0}=544.9 \mathrm{meV}(284.8 \mathrm{meV}), D=4.747 \mathrm{eV}$ [32] $(2.648 \mathrm{eV}), I=I_{1 \sigma_{g}}=15.427 \mathrm{eV}, \Gamma=0.05 \mathrm{eV}$. NO $X^{2} \Pi$ $\left(\mathrm{NO}^{+}\left(1 s_{O}^{-1}\right)\right): R_{0}=2.1754$ a.u. [31] (2.2495 a.u. [33]), $\omega_{0}=$ $236 \mathrm{meV}$ [31] $(218 \mathrm{meV}$ [33]), $D=6.6 \mathrm{eV} \mathrm{[31]}(5.4303 \mathrm{eV}$ [34]), $I=I_{O 1 s} \approx 543.5 \mathrm{eV}$ [35], $\Gamma=0.085 \mathrm{eV}$ [33]. The values of $\left(R_{0}^{(i)}, \omega_{0}^{(i)}, D_{i}\right)$ in brackets are for the ionized state. The large $\Gamma=0.05 \mathrm{eV}$ used for $\mathrm{H}_{2}$ does not affect the results due to the larger Doppler broadening. We used in the simulations $\chi=145^{\circ}$, which is a possible setup in photoelectron spectroscopy and also because the recoil effect is enhanced for this angle with respect to $\chi=90^{\circ}$ (see Fig. 2 and the discussion below). However, as we see below, due to the large Doppler broadening the best way to observe the discussed effect is the detection of the fragment of recoil-induced dissociation. In this case we should integrate the cross section over all angles $\chi$ of ejection of the photoelectron with respect to the photon momentum. This integration is not important for hydrogen molecules (Fig. 4) because the $\chi$ dependence becomes significant only for $\omega \gtrsim 10 \mathrm{keV}$ (see Fig. 2).

The semiclassical Hamiltonian (8) sheds light on the qualitatively different vibrational and rotational recoil-induced dissociations. Although Eq. (7) shows that when angle $\theta$ is arbitrary one cannot separate the vibrational and rotational recoil effects, deeper insight can be reached by considering two representative angles, $\theta=0^{\circ}$ and $\theta=90^{\circ}$. In the first case we have a pure vibrational dissociation while in the second one, rotational dissociation. Both vibrational and rotational recoil effects lead to the dissociation for intermediate angles $\theta$.

First, consider the role of the recoil effect in the ionization profile of the hydrogen molecule. The formal reason for the difference between vibrational and rotational recoil effects is the recoil factor $\exp (l \alpha q R \cos \theta)$ in the FC amplitude (6). This factor being equal to $\exp (l \alpha q R)$ for the purely vibrational recoil effect $\left(\theta=0^{\circ}\right)$ experiences fast oscillations which are compensated by the fast oscillations of the nuclear wave function resulting in the nuclear momentum $-\alpha q$ and rather high nuclear kinetic energy $E_{\text {kin }}=E_{\text {rec }}^{\mathrm{vib}}\left(\theta=0^{\circ}\right) \approx 3.2 \mathrm{eV}$ in the point of vertical transition [Fig. 3(a)]. As a result, the molecule starts almost instantaneously to dissociate. In contrast, the recoil factor $\exp (\iota \alpha q R \cos \theta)=1$ for the purely rotational recoil effect $\left(\theta=90^{\circ}\right)$. Thus, now we have the ordinary FC amplitude where the vertical transition ends up in the classical turning point with zero nuclear velocity [Fig. 3(b)]. But contrary to the former case this vertical transition occurs in the effective potential $V_{i}\left(R, \theta=90^{\circ}\right)$, which is strongly lifted up by the centrifugal potential [Fig. 3(b)]. Now the molecule starts the dissociation slowly from velocity $u=0$. Due to the centrifugal force the molecule is accelerated along the interatomic coordinate causing the bond to break 

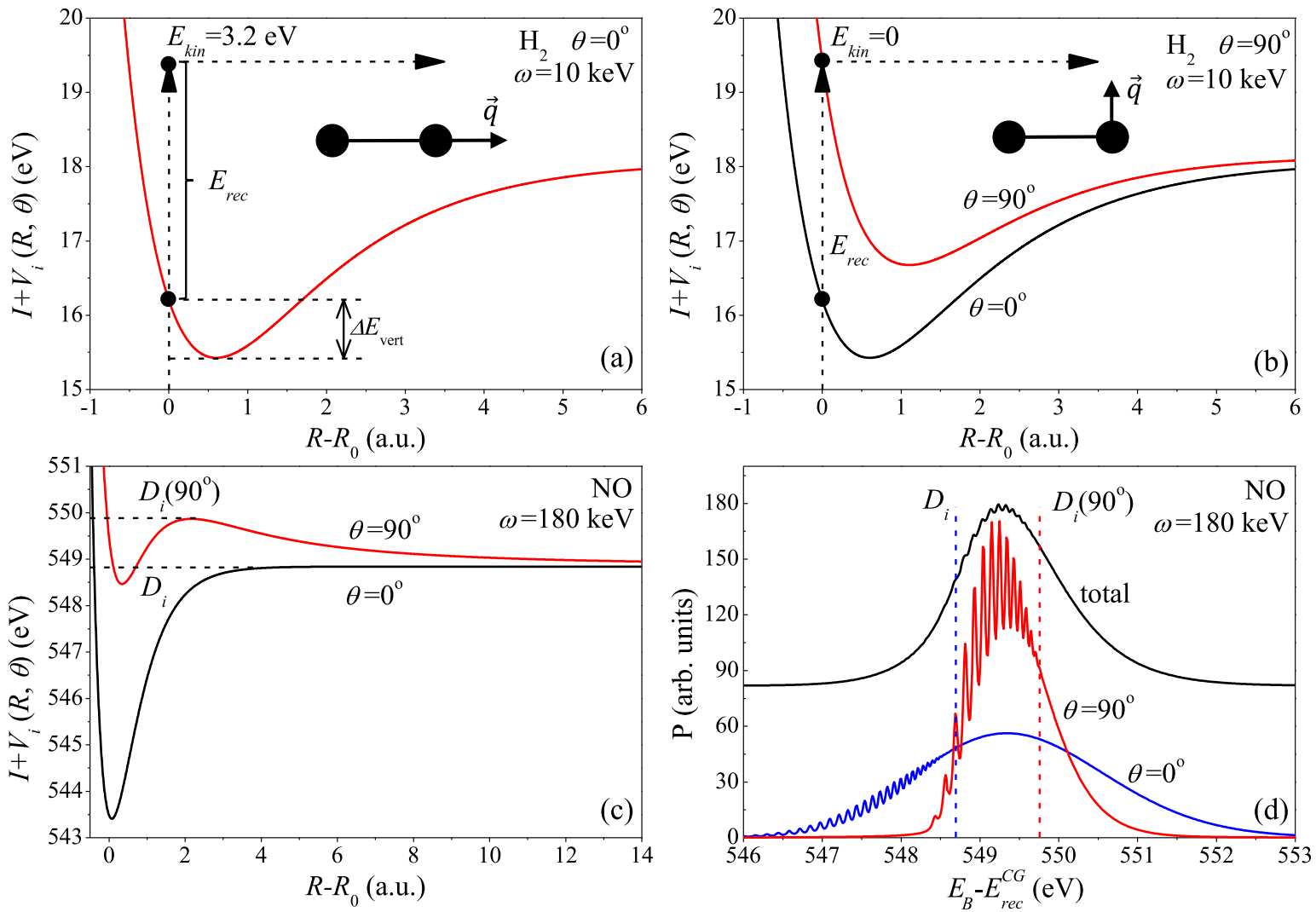

FIG. 3. Physical picture of the vibrational $\left(\theta=0^{\circ}\right)$ and rotational $\left(\theta=90^{\circ}\right)$ recoil-induced dissociation in the course of the $1 \sigma_{g}$ ionization of the $\mathrm{H}_{2}$ molecule and $\mathrm{O} 1 s$ ionization of the NO molecule. $D_{i}(\theta)$ is the dissociation energy of the effective potential $V_{i}(R, \theta)$ [Eq. (8)]. For better visibility the total probability $P\left(E_{B}, \omega, \chi\right)$ (shown by black line) is lifted up. $\chi=145^{\circ}$.

[Fig. 3(b)]. The spectral shape of the probability of ionization is shown in Fig. 4. In spite of the different physics behind the vibrational and rotational recoil effects, the profiles for $\theta=0^{\circ}$ and $90^{\circ}$ are very similar [Fig. 4(a)]. One should notice that in the energy range $\omega \leqslant 5 \mathrm{keV}$ the electronic recoil effect dominates and the role of relativistic effects is weak contrary to the region $\omega \gtrsim 10 \mathrm{keV}$ [Fig. 4(b)] where the nonrelativistic approximation is not valid anymore. One can see that the recoil-induced dissociation starts to take place from rather low photon energy $(\omega \gtrsim 5 \mathrm{keV})$. The reason for this is the small mass of the hydrogen and the low dissociation energy in the ionized state, $D_{i}=2.648 \mathrm{eV}$. One should mention that in the simulations we have neglected the coherent ejection of the photoelectron from both hydrogen atoms of $\mathrm{H}_{2}$ which results in the Cohen-Fano interference [5-7,14,36,37]. This is legitimate since this interference is quenched for the case studied here of hard-x-ray photon energies $[5,36]$.

It is interesting to notice that the peak position of the rotational cross section $\left(\theta=90^{\circ}\right)$ is red shifted in comparison with the case $\theta=0^{\circ}$ [see Fig. 4(a)], The reason for this shift is that the maximum of the FC factor for boundcontinuum transition is shifted by $\delta=F_{i} a_{i}$ with respect to the vertical transition [38]. Here $F_{i}=\partial V_{i}(R, \pi / 2) /\left.\partial R\right|_{R=R_{0}}$ and $a_{i}=\left(2 \mu F_{i}\right)^{-1 / 3}$. For example $\delta=1.7 \mathrm{eV}$ for $\omega=30 \mathrm{keV}$ in good agreement with observed shift in Fig. 4(a). The spectra obtained with the commonly used experimental detection angle of $\chi=90^{\circ}$ are compared with the spectra for $\chi=145^{\circ}$ in Fig. 4(c). One can see that there is a red shift of the maximum of the probability for $\chi=90^{\circ}$ with respect to $\chi=145^{\circ}$, which reflects the larger momentum $q$ transferred to the molecule for $\chi=145^{\circ}$, as previously shown in Fig. 2.

The picture changes drastically in the case of the O1s photoionization of the NO molecule. Here, the recoil-induced dissociation starts to occur from the energy $\omega=200 \mathrm{keV}$ (Fig. 5) which is rather close to the rest energy of the photoelectron (see Fig. 2). This makes both photon and electron recoils important as well as the relativistic effects (Fig. 5). Due to the higher dissociation energy $D_{i}$ for NO than for $H_{2}$, the effective potential $V_{i}(R, \theta)$ for $\theta=90^{\circ}$ has a strong barrier which shifts up the dissociation energy $D_{i}\left(90^{\circ}\right)$ [Fig. 3(c)]. Therefore the rotational recoil effect needs larger $E_{\text {rec }}$ to overcome $D_{i}\left(90^{\circ}\right)$. This results in a blue shift of the dissociation threshold for $\theta=90^{\circ}$ in comparison with $\theta=0^{\circ}$ [Fig. 3(d)].

The FC amplitude (6) can be computed analytically for a harmonic oscillator for fixed angle $\theta$ and $\omega_{0}=\omega_{0}^{(i)}$ to find the probability $P_{0 v}(\theta)=\left|F_{0 v}\right|^{2}$ of vibrational excitation

$$
P_{0 v}(\theta)=e^{-S(\theta)} \frac{S^{\nu}(\theta)}{\nu !} .
$$

The two qualitatively different contributions to the HuangRhys (HR) parameter $S(\theta)=S_{\text {shift }}+S_{\text {rec }}^{\mathrm{vib}}(\theta)$ allow us to identify two sources of the vibrational excitation. The first one $S_{\text {shift }}=x_{0}^{2} / 2 a^{2}=\Delta E_{\text {vert }} / \omega_{0}$ is due to the shift $x_{0}=R_{0}^{(i)}-R_{0}$ 

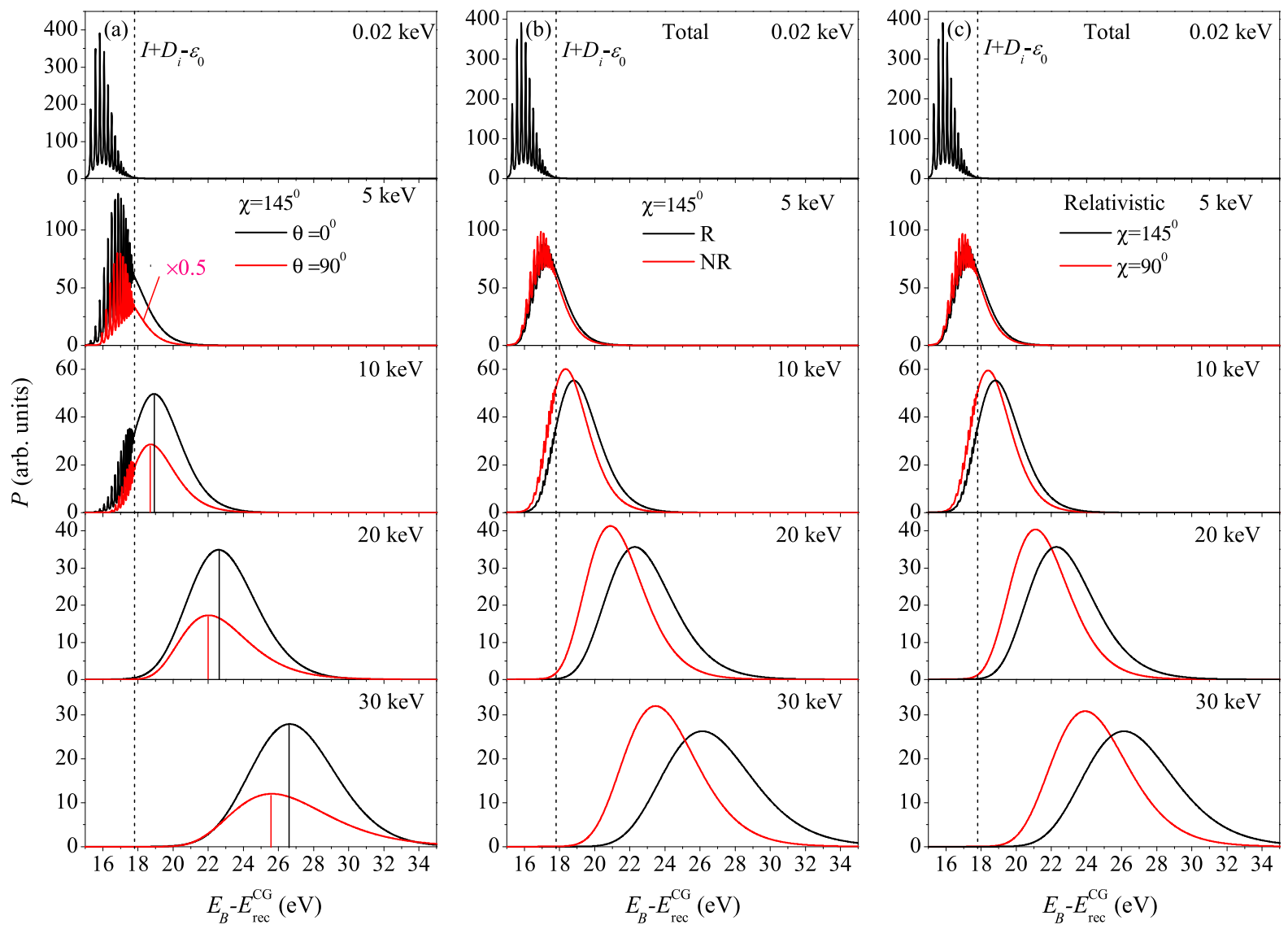

FIG. 4. Photoelectron spectra of the $\mathrm{H}_{2}$ molecule. (a) The semiclassical probability (7) $P\left(E_{B}, \omega, \chi, \theta\right)$ for $\theta=0^{\circ}$ (vibrational recoil) and $90^{\circ}$ (rotational recoil), $\theta=\angle(\mathbf{q}, \mathbf{R})$. The dashed vertical line shows the dissociation limit, and the solid vertical lines show the peak positions of the probabilities. For better visibility, all probabilities $P$ for $\theta=90^{\circ}$ in 1 (a) are scaled by a factor of 0.5 . (b) The total probability $P\left(E_{B}, \omega, \chi\right)$ computed using the relativistic equation (2) for the recoil momentum $q$ and the nonrelativistic one $q \approx \sqrt{2 m \Omega}$ marked as (R) and (NR), respectively. One can see that the relativistic effects become important starting from $\omega \approx 10 \mathrm{keV}$. (c) The total probabilities $P\left(E_{B}, \omega, \chi\right)$ for $\chi=90^{\circ}$ and $\chi=145^{\circ}$ computed using the relativistic equation (2).

of the minima position $R_{0}^{(i)}$ of the potential $V_{i}(R)$ of coreionized state with respect to $R_{0}$, where $\Delta E_{\text {vert }}$ is the energy of the vertical transition with respect to $\left[V_{i}(R)\right]_{\min }=0$. The second reason is the vibrational recoil along the molecular axis $S_{\mathrm{rec}}^{\mathrm{vib}}(\theta)=(q \alpha a \cos \theta)^{2} / 2=E_{\mathrm{rec}}^{\mathrm{vib}}(\theta) / \omega_{0}$. Here $a=1 / \sqrt{\mu \omega_{0}}$ and $\omega_{0}$ is the vibrational frequency. This explains the increase of the intensity of higher vibrational levels with increase of $\omega$ (see insert in Fig. 5). Taking into account that the total recoil energy $E_{\text {rec }}$ is the sum of the energies of vibrational and rotational recoils (5) one can include the rotational recoil effect in the probability $P_{0 v}$ by simple replacement

$$
S(\theta) \rightarrow S=\frac{\Delta E_{\mathrm{vert}}+E_{\mathrm{rec}}}{\omega_{0}} .
$$

This equation explains the physical meaning of the HR parameter $S$ which is the effective quantum number of vibrational level which is mostly populated in the course of photoionization. In spite of this crude approximation, Eq. (10) gives a simple semiquantitative description of the studied rovibrational excitation. One should notice that contrary to the Poisson distribution (9) which is valid only for a harmonic potential, its asymptote $P_{0 v} \approx(2 \pi S)^{-1 / 2} \exp \left[-(\nu-S)^{2} / 2 S\right]$ for $S \gg 1$ is valid for any potential shape [39]. The Gaussian distribution allows us to write the energy-normalized probability of the photoionization as

$$
P\left(E_{B}\right) \approx \frac{1}{\Delta \sqrt{\pi}} \exp \left(-\frac{\left(E_{B}-I-E_{\max }\right)^{2}}{\Delta^{2}}\right),
$$

where $E_{\mathrm{max}}=\Delta E_{\mathrm{vert}}+E_{\mathrm{rec}}+E_{\mathrm{rec}}^{\mathrm{CG}}$ is the peak position and $\Delta_{\mathrm{FWHM}}=\Delta \sqrt{4 \ln 2}=\omega_{0} \sqrt{8 S \ln 2}$ is the full width at half maximum. Equation (11) says that the molecule will dissociate whenever the recoil energy is high enough that $E_{\max }$ exceeds the dissociation energy $D_{i}$ of the ionized molecule: $\Delta E_{\text {vert }}+E_{\text {rec }}>D_{i}$. It is interesting to notice that the peak position given by this equation $E_{B}-E_{\mathrm{rec}}^{\mathrm{CG}}=I+E_{\mathrm{max}} \approx I+$ $E_{\text {rec }}$ nicely coincides with the $a b$ initio calculation of the peak position of $P\left(E_{B}, \omega, \chi\right)$ calculated using Eq. (7). For example $I+E_{\text {rec }}=26.398,22.342$, and $18.643 \mathrm{eV}$ for $\mathrm{H}_{2}$ is very close to the peak position of $P\left(E_{B}, \omega, \chi\right)$ (7) 26.16, 22.29 , and $18,8 \mathrm{eV}$ for $\omega=30,20$, and $10 \mathrm{keV}$, respectively [see Fig. 4(b)].

One should notice that the translational and rotational Doppler broadening $D_{\mathrm{dop}}=D_{\mathrm{dop}}^{\mathrm{tr}}+D_{\mathrm{dop}}^{\text {rot }} \approx q \bar{v}\left(1+2 M_{B} / 3 M_{A}\right)$ [11] is significant for ambient conditions in the high-energy region. For example $D_{\mathrm{dop}} \approx 1.2 \mathrm{eV}(0.96 \mathrm{eV})$ for the NO $\left(\mathrm{H}_{2}\right)$ molecule at $T=300 \mathrm{~K}, \omega=150 \mathrm{keV}(10 \mathrm{keV})$, and $\chi=145^{\circ}\left(\bar{v}=\sqrt{2 k_{B} T / M}\right)$. Large broadening caused by the 


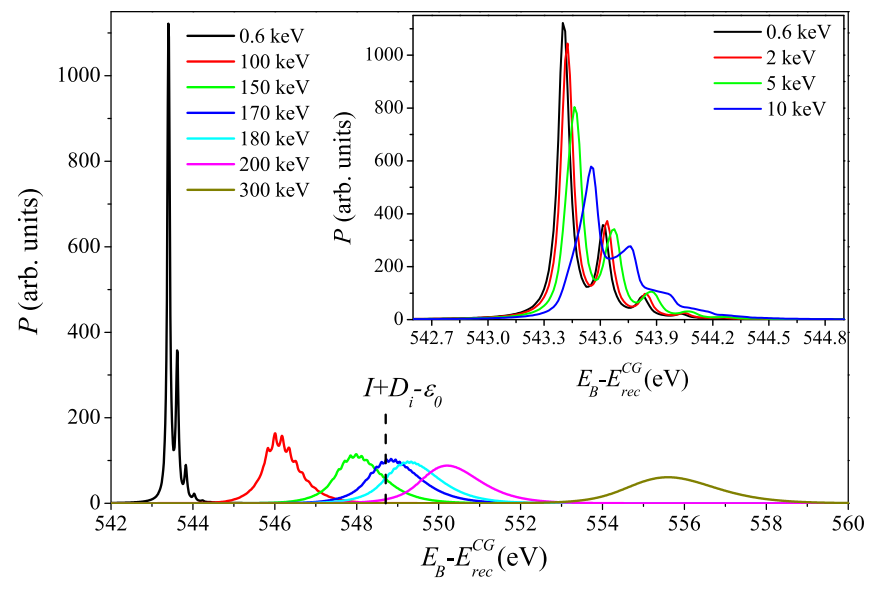

FIG. 5. Total semiclassical probabilities (7) $P\left(E_{B}, \omega, \chi\right)$ of $\mathrm{O} 1 s$ ionization of NO molecule. The vertical line shows the dissociation limit. $\theta=\angle(\mathbf{q}, \mathbf{R})$. The insert shows the recoil-induced vibrational excitation for $\omega$ below dissociation threshold. $\chi=145^{\circ}$.

Doppler effect does not allow us to resolve the vibrational structure. This washes out the boundary between boundbound and bound-continuum transitions (Fig. 4) which immediately evidences the dissociation. This hinders the direct observation of the recoil-induced dissociation in the photoelectron spectrum. Figure 5 shows the recoil-induced blue shift of the maximum of the photoelectron line with the increase of $\omega$. However, the recoil-induced dissociation starts only when this shift exceeds the dissociation energy of the core-ionized state $D_{i}$ (Fig. 5). Thus, we need to know $D_{i}$ to evidence the recoil-induced dissociation. Nevertheless, there is an alternative and direct way to observe this dissociation. One can measure directly the fragments of the recoil-induced dissociation in the time-of-flight mode. The fingerprint of the recoil-induced fragmentation in this case is given by the $\omega$ dependence of the kinetic energy of the fragment of dissociation.

\section{CONCLUSION}

The discussed effect can be observed directly for the $\mathrm{H}_{2}$ molecule by measuring the high-energy photoelectron spectra of the $\mathrm{H}_{2}$ molecule $\left(D_{i}=2.648 \mathrm{eV}\right)$ at the SOLEIL synchrotron [20] and SACLA XFEL [24] or by detecting the $\omega$ dependence of the velocity of $\mathrm{H}^{+}$ions using timeof-flight spectroscopy. In the case of the NO molecule the recoil-induced dissociation can be observed at the PETRA synchrotron using time-of-flight spectroscopy for dissociation ions as well by detecting the atomic peak in fluorescence
[40] or in Auger spectra [41] or optical fluorescence as in Refs. [28,29]. It is appropriate to notice that the recoil-induced dissociation can be studied also using the recoil-induced Doppler splitting of the resonance in the Auger decay process [16]. Furthermore, our preliminary analysis shows that the effect can be observed also in hard-X-ray F1s ionization of $\mathrm{CF}_{4}$, $\mathrm{PF}_{5}, \mathrm{SF}_{6}$, and $\mathrm{MoF}_{6}$ molecules. According to Ref. [42] the $\mathrm{F} 1 s$ ionization results in the dissociation of these molecules. The discussed recoil effect increases the kinetic energy of fragments of dissociation. We suggest to investigate the role of the recoil effect by measuring the growth of the kinetic energy of the fragments of dissociation with the increase of $\omega$. It is important to notice that there is a threshold for the recoil-induced dissociation when the core-ionized state is bound (see Fig. 3): $E_{\text {rec }} \gtrsim D_{i}$. However, this effect does not have a threshold for the discussed molecules because the core-ionized state is dissociative. Preliminary estimations for the $\mathrm{CF}_{4}$ molecule show that the recoil-induced increase of the kinetic energy of the dissociation fragment could be detected for photon energies below $100 \mathrm{keV}$. One should point out also that in the case of polyatomic molecules the recoil energy will be distributed between different nuclear degrees of freedom.

The discussed mechanism of dissociation can be observed also for surface adsorbed molecules or for surface atoms. In spite that the main part of the hard-x-ray photons will be absorbed by the bulk atoms, some part of the surface atoms will be also ionized. To increase the amount of signal from the surface one can use the low grazing angle set-up. This direction of investigation can be important for surface sciences which need information about the strength of the chemical bond on the surface layer.

\section{ACKNOWLEDGMENTS}

We thank Marc Simon and Renaud Guillemin for fruitful discussions of the experimental observation of the effect studied here. This work is supported by the Swedish Research Council (VR). J.-C.L. thanks the support by the National Science Foundation of China under Grants No. 11574082 and No. 11974108 and the Fundamental Research Funds for the Central Universities (No. 2018MS050). F.G. and S.P. acknowledge support within the State contract of the Ministry of Education and Science of the Russian Federation for Siberian Federal University for Scientific Research in 20172019 (Project No. 3.2662.2017); V.V.C. and A.F. acknowledge funding from the ERC-ADG-2014 - Advanced Investigator Grant No. 669531 EDAX under the Horizon 2020 EU Framework, Programme for Research and Innovation.
[1] W. Demtröder, Laser Spectroscopy. Basic Concepts and Instrumentation (Springer-Verlag, Berlin, 2003).

[2] F. Kh. Gel'mukhanov, L. N. Mazalov, and N. A. Shklyaeva, Zh. Eksp. Teor. Fiz. 71, 960 (1976) [Sov. Phys. JETP 44, 504 (1976)].
[3] W. Domcke and L. S. Cederbaum, J. Electron. Spectrosc. Relat. Phenom. 13, 161 (1978).

[4] V. C. Felicíssimo, F. F. Guimarães, and F. Gel'mukhanov, Phys. Rev. A 72, 023414 (2005).

[5] X.-J. Liu, N. A. Cherepkov, S. K. Semenov, V. Kimberg, F. Gel'mukhanov, G. Prümper, T. Lischke, T. Tanaka, M. 
Hoshino, H. Tanaka, and K. Ueda, J. Phys. B: At. Mol. Opt. Phys. 39, 4801 (2006).

[6] K. Ueda, X.-J. Liu, G. Prümper, T. Lischke, T. Tanaka, M. Hoshimo, H. Tanaka, I. Minkov, V. Kimberg, and F. Gel'mukhanov, Chem. Phys. 329, 329 (2006).

[7] K. Ueda, R. Püttner, N. A. Cherepkov, F. Gel'mukhanov, and M. Ehara, Eur. Phys. J.: Spec. Top. 169, 95 (2009).

[8] E. Kukk, T. D. Thomas, D. Céolin, S. Granroth, O. Travnikova, M. Berholts, T. Marchenko, R. Guillemin, L. Journel, I. Ismail, R. Püttner, M. N. Piancastelli, K. Ueda, and M. Simon, Phys. Rev. Lett. 121, 073002 (2018).

[9] T. D. Thomas, E. Kukk, H. Fukuzawa, K. Ueda, R. Püttner, Y. Tamenori, T. Asahina, N. Kuze, H. Kato, M. Hoshino, H. Tanaka, M. Meyer, J. Plenge, A. Wirsing, E. Serdaroglu, R. Flesch, E. Rühl, S. Gavrilyuk, F. Gel'mukhanov, A. Lindblad, and L. J. Saethre, Phys. Rev. A 79, 022506 (2009).

[10] D. Céolin, J.-C. Liu, V. Vaz da Cruz, H. Ågren, L. Journele, R. Guillemin, T. Marchenko, R. K. Kushawaha, M. N. Piancastelli, R. Püttner, M. Simon, and F. Gel'mukhanov, Proc. Natl. Acad. Sci. USA 116, 4877 (2019).

[11] Y.-P. Sun, C.-K. Wang, and F. Gel'mukhanov, Phys. Rev. A 82, 052506 (2010).

[12] T. D. Thomas, E. Kukk, K. Ueda, T. Ouchi, K. Sakai, T. X. Carroll, C. Nicolas, O. Travnikova, and C. Miron, Phys. Rev. Lett. 106, 193009 (2011).

[13] C. Miron, Q. Miao, C. Nicolas, J. D. Bozek, W. Andrałojć, M. Patanen, G. Simões, O. Travnikova, H. Ågren, and F. Gel'mukhanov, Nat. Commun. 5, 3816 (2014).

[14] Q. Miao, O. Travnikova, F. Gel'mukhanov, V. Kimberg, Y.-P. Sun, T. D. Thomas, C. Nicolas, M. Patanen, and C. Miron, J. Phys. Chem. Lett. 6, 1568 (2015).

[15] S. Gavrilyuk, Y.-P. Sun, S. Levin, H. Ågren, and F. Gel'mukhanov, Phys. Rev. A 81, 035401 (2010).

[16] M. Simon, R. Püttner, T. Marchenko, R. Guillemin, R. K. Kushawaha, L. Journel, G. Goldsztejn, M. N. Piancastelli, J. M. Ablett, J.-P. Rueff, and D. Céolin, Nat. Commun. 5, 4069 (2014).

[17] Y. Takata, Y. Kayanuma, M. Yabashi, K. Tamasaku, Y. Nishino, D. Miwa, Y. Harada, K. Horiba, S. Shin, S. Tanaka, E. Ikenaga, K. Kobayashi, Y. Senba, H. Ohashi, and T. Ishikawa, Phys. Rev. B 75, 233404 (2007).

[18] S. Suga, A. Sekiyama, H. Fujiwara, Y. Nakatsu, T. Miyamachi, S. Imada, P. Baltzer, S. Niitaka, H. Takagi, K. Yoshimura, M. Yabashi, K. Tamasaku, A. Higashiya, and T. Ishikawa, New J. Phys. 11, 073025 (2009).

[19] Y. Takata, Y. Kayanuma, S. Oshima, S. Tanaka, M. Yabashi, K. Tamasaku, Y. Nishino, M. Matsunami, R. Eguchi, A. Chainani, M. Oura, T. Takeuchi, Y. Senba, H. Ohashi, S. Shin, and T. Ishikawa, Phys. Rev. Lett. 101, 137601 (2008).

[20] J. P. Rueff, J. M. Albett, D. Céolin, D. Prieur, T. Moreno, V. Baléndet, B. Lassale-Kaiser, J. E. Raullt, M. Simon, and A. Shukla, J. Synchrotron Radiat. 22, 175 (2015).

[21] Y. Takata, M. Yabashi, K. Tamasaku, Y. Nishino, D. Miwa, T. Ishikawa, E. Ikenaga, K. Horiba, S. Shin, M. Arita, K. Shimada, H. Namatame, M. Taniguchi, H. Nohira, T. Hattori,
S. Södergren, B. Wannberg, and K. Kobayashi, Nucl. Instrum. Methods Phys. Res. A 547, 50 (2005).

[22] H. Franz, O. Leupold, R. Röhlsberger, S. V. Roth, O. H. Steck, J. Spengler, J. Strümpfen, M. Fischer, J. Viefhaus, E. Meckert, and T. Wroblewski, Synchrotron Rad. News 19, 25 (2006).

[23] A. Burkhardt, T. Pakendorf, B. Reime, J. Meyer, P. Fischer, N. Stübe, S. Panneerselvam, O. Lorbeer, K. Stachnik, M. Warmer, P. Rödig, D. Göries, and A. Meents, Eur. Phys. J. Plus 131, 56 (2016).

[24] M. Yabashi, H. Tanaka, and T. Ishikawa, J. Synchrotron Rad. 22, 477 (2015).

[25] F. Gel'mukhanov and H. Ågren, Phys. Rep. 312, 87 (1999).

[26] P. Morin and C. Miron, J. Electr. Spectr. Relat. Phenom. 185, 259 (2012).

[27] D. Céolin, J. M. Ablett, D. Prieur, T. Moreno, J. P. Rueff, T. Marchenko, L. Journel, R. Guillemin, B. Pilette, T. Marin, and M. Simon, J. Electr. Spectr. Relat. Phenom. 190, 188 (2013).

[28] H. C. Choi, R. M. Rao, A. G. Mihill, S. Kakar, E. D. Poliakoff, K. Wang, and V. McKoy, Phys. Rev. Lett. 72, 44 (1994).

[29] E. D. Poliakoff, H. C. Choi, R. M. Rao, A. G. Mihill, S. Kakar, K. Wang, and V. McKoy, J. Chem. Phys. 103, 1773 (1995).

[30] V. B. Berestetskii, E. M. Lifshitz, and L. P. Pitaevskii, Quantum Electrodynamics, Course of Theoretical Physics (Elsevier, Singapore, 2008), Vol. 4.

[31] K. P. Huber and G. Herzberg, Molecular Spectra and Molecular Structure IV. Constants of Diatomic Molecules (Van Nostrand Reinhold, New York, 1979).

[32] W. Kolos and C. C. J. Roothan, Rev. Mod. Phys. 32, 219 (1960).

[33] R. Püttner, I. Dominguez, T. J. Morgan, C. Cisneros, R. F. Fink, E. Rotenberg, T. Warwick, M. Domke, G. Kaindl, and A. S. Schlachter, Phys. Rev. A 59, 3415 (1999).

[34] A. Andersen and Y. Öhrn, J. Mol. Spectrosc. 45, 358 (1973).

[35] K. Siegbahn, C. Nordling, G. Johansson, J. Hedman, P. F. Hedén, K. Hamrin, U. Gelius, T. Bergmark, L. O. Werme, R. Manne, and Y. Baer, in ESCA Applied to Free Molecules (North-Holland, Amsterdam, 1969), p. 75.

[36] H. D. Cohen and U. Fano, Phys. Rev. 150, 30 (1966).

[37] F. Kh. Gel'mukhanov, L. N. Mazalov, and N. A. Shklyaeva, Zh. Eksp. Teor. Fiz. 69, 1971 (1975) [Sov. Phys. JETP 42, 1001 (1976)].

[38] F. Gel'mukhanov and H. Ågren, Phys. Rev. A 54, 379 (1996).

[39] V. C. Felicíssimo, I. Minkov, F. F. Guimarães, F. Gel'mukhanov, A. Cesar, and H. Ågren, Chem. Phys. 312, 311 (2005).

[40] A. Pietzsch, Y.-P. Sun, F. Hennies, Z. Rinkevicius, H. O. Karlsson, T. Schmitt, V. N. Strocov, J. Andersson, B. Kennedy, J. Schlappa, A. Föhlisch, J.-E. Rubensson, and F. Gel'mukhanov, Phys. Rev. Lett. 106, 153004 (2011).

[41] O. Björneholm, M. Bässler, A. Ausmees, I. Hjelte, R. Feifel, H. Wang, C. Miron, M. N. Piancastelli, S. Svensson, S. L. Sorensen, F. Gel'mukhanov, and H. Ågren, Phys. Rev. Lett. 84, 2826 (2000).

[42] A. G. Falaleev, V. A. Andreev, and V. I. Vovna, Int. J. Quantum Chem. 43, 573 (1992). 